\title{
A DINÂMICA DOS MODELOS DE NEGÓCIOS PARA O AGRONEGÓCIO: UMA VISÃO BIBLIOMÉTRICA DE 1956 A 2017 PELA WEB OF SCIENCE
}

\author{
João Paulo Nascimento da Silva ${ }^{1}$ \\ Cledison Carlos de Oliveira ${ }^{2}$ \\ André Spuri Garcia ${ }^{3}$
}

\begin{abstract}
RESUMO
O agronegócio se apresenta como importante fator para economia brasileira e global, sendo assim, este fenômeno se mostra importante para aprofundamento das pesquisas acadêmicas por parte de seu desenvolvimento como modelo de negócios. Através desta pesquisa bibliométrica é possível aprofundar sobre o desenvolvimento deste fenômeno na academia, que, conforme apontam os dados, tem se mostrado de grande interesse para economias como a Brasil e EUA, e que as pesquisas sobre esta temática caminham próximo ao pensamento da economia destes países, conforme apontado pelo apoio dado as fontes de fomento. Dessa forma, este estudo apresenta o fenômeno crescente na economia global, através da pesquisa via Web of Science, e que acompanha o mesmo como desenvolvimento econômico.
\end{abstract}

Palavras-chave: Comportamento da academia, economia, produção.

\section{BUSINESS MODELS FOR AGRIBUSINESS: A BIBLIOMETRIC VIEW OF 1956 TO 2017 IN WEB OF SCIENCE DATABASE}

\begin{abstract}
Agribusiness is an important factor for the Brazilian and global economy, thus, this phenomenon is important for the deepening of academic research by its development as a business model. Through this bibliometric research it is possible to deepen the development of this phenomenon in academia, which, as the data point out, has been of great interest to economies such as Brazil and the USA, and that research on this subject is close to the thinking of their economics. countries, as indicated by the support given to funding sources. Thus, this study presents the growing phenomenon in the global economy, through research via Web of Science, and accompanying it as economic development.
\end{abstract}

Key words: Academy behavior, economics, production.

\footnotetext{
${ }^{1}$ Graduado em Administração (FIVJ). Mestre em Marketing Relacional (IPL/PT). Mestre em Administração (UFLA). Doutorando em Administração (UFLA). E-mail: jpnsilvas@gmail.com.

2 Graduado em Contabilidade (Fadminas). Mestre em Administração (UFLA). Doutorando em Administração (UFLA). E-mail: oliveira.cledison@gmail.com.

${ }^{3}$ Graduado em Administração (UFOP). Mestre em Administração (UFLA). Doutorando em Administração

(UFLA). Professor na Universidade do Estado de Minas Gerais (UEMG). E-mail: andrespurigarcia@gmail.com.
} 


\section{INTRODUÇÃO}

O agronegócio é um importante fator do desenvolvimento econômico do Brasil e um importante traçado para economia mundial. Segundo Arbage (2012) o agronegócio abrange toda a produção agropecuária (que inclui as produções animal e vegetal, atividades vinculadas ao extrativismo e a exploração florestal), bem como as atividades situadas à montante da produção rural (indústrias de insumos, estrutura de financiamento à produção, instituições de pesquisa, etc.) e à jusante como é o caso da indústria de processamento, do setor de distribuição (atacado e varejo) e dentre outras empresas e/ou organizações que contribuem para o processo de transformação e comercialização de produtos agropecuários.

Nesse sentido, o desenvolvimento de modelos de negócios voltados para o agronegócio contempla um importante ponto da academia mundial em relação ao desenvolvimento deste fenômeno. Em relação ao Brasil, o país demonstra, através da participação das pesquisas brasileiras, o importante passo de nosso desenvolvimento acadêmico e econômico.

Para Veldwisch (2015), os modelos de negócios voltados para o agronegócio trazem mudanças no campo, de forma a melhorar suas implementações e relações com o ambiente. Segundo Verdouw (2015), os negócios do campo devem se adaptar de forma flexível às condições impostas pelas mudanças do mercado.

Apresentar uma definição de modelo de negócio

A partir desse pensamento, busca-se compreender o fenômeno dos modelos de negócios voltados para agricultura, sendo um fator impactante em economias e nos fatores de desenvolvimento local, regional, de empresas e nações, e que surgem como fator de relevância para a sustentabilidade dos negócios no longo prazo. Dessa forma, é importante buscar compreender o fenômeno de modelos de negócios voltados para mercados agropecuários contemporâneos, pois o modelo de negócios afeta seu desenvolvimento e, consequentemente, afeta os resultados das pesquisas e da economia como um todo.

O objetivo deste artigo é mapear o estado da pesquisa em desenvolvimento de modelo de negócios para o agronegócio, a fim de proporcionar uma melhor compreensão da estrutura deste campo de pesquisa e dos principais atores envolvidos. Especificamente, procura-se apresentar as tendências de publicação nesse campo, as categorias (áreas) das publicações, as palavras chaves e países centrais, os artigos, periódicos e autores mais relevantes, as estruturas de cocitação e coautoria dos mesmos.

Nesse sentido, através da bibliometria, este estudo busca apresentar uma avaliação das atividades de produção acadêmica e comunicação da comunidade científica (ARAÚJO, 2006), assim como contribuir com o referido campo de conhecimento, de forma a mapear as comunidades acadêmicas, e identificar grupos de pesquisadores e motivações para desenvolvimento das pesquisas, de forma a entender este comportamento da academia como um todo (CHUEKE; AMATUCCI, 2015, p. 2).

Para tanto, este estudo visa compreender o fenômeno do desenvolvimento de modelos de negócios para o agronegócio, aplicando uma pesquisa da produção acadêmica via Web of Science dos anos de 1956, onde foi encontrado o primeiro artigo sobre a temática, até o ano de 2017, com intuito de processar e compreender o comportamento da academia em relação a estas pesquisas e a participação da academia brasileira em relação a produção global. Esta análise pode gerar insights sobre o fenômeno do desenvolvimento econômico de alguns países que mais se aprofundam nesta relação de pesquisas sobre os modelos de negócios para o agronegócio. 
Este artigo foi dividido em etapas que consistem, a seguir, na apresentação e aprofundamento da literatura sobre a temática de modelos de negócios e agronegócios, seguida pelo componente de embasamento metodológico em que a pesquisa foi realizada, e, na terceira etapa, a apresentação e discussão dos resultados encontrados sobre o direcionamento das pesquisas acadêmicas, seguida pelas conclusões e pelas sugestões para pesquisas futuras.

\section{REFERENCIAL TEÓRICO}

Segundo Osterwalder (2004), o modelo de negócios consiste na lógica de criação, entrega e captura de valor de uma empresa. A introdução de modelos de negócios no agronegócio, provoca mudanças no campo, transformando a prática econômica implementando novos produtos, serviços, melhores processos, bem como, a implementação de um novo método de organização do trabalho e suas relações com o meio ambiente (VELDWISCH, 2015). Nesse sentido, Verdouw, et al. (2015) salienta que atualmente a agricultura é uma tarefa gerencial complexa que impõe requisitos rigorosos na fazenda, como Sistemas de Gerenciamento de Informação. Embora exista uma extensa literatura sobre a teoria de modelos de negócios para fornecer exemplos de empresas específicas, não existe uma visão abrangente de como as empresas devem se aproximar incorporando a sustentabilidade em seus modelos comerciais (BAINES et al., 2007).

Sommer (2012) enfatiza que um modelo de negócios não só tem foco na empresa, mas envolve um conjunto mais amplo de partes interessadas, exigindo uma perspectiva de rede de valor mais ampla para inovar e transformar o modelo de negócio. Isso está de acordo com Beattie e Smith (2013) e Zott, Amit e Massa (2011) que descrevem como modelo de negócio que se estende além da entidade da empresa, seus clientes e acionistas, e incluindo o valor capturado para as principais partes interessadas. Da mesma forma, os modelos de negócios sustentáveis capturam valor econômico, social e ambiental para uma ampla gama de stakeholders (BOCKEN et. al., 2013).

Os modelos de negócios sustentáveis incorporam uma abordagem de linha tripla e consideram uma ampla gama de interesses das partes interessadas, incluindo o meio ambiente e a sociedade. Eles são importantes em conduzir e implementar a inovação corporativa para a sustentabilidade, pode ajudar a incorporar a sustentabilidade na finalidade e nos processos comerciais, e servem de agente principal da vantagem competitiva (BOCKEN et. al., 2014).

Nessa perspectiva as empresas multinacionais de alimentos e agronegócios estão redesenhando suas estratégias de abastecimento para aproveitar o potencial de produção de alimentos subutilizados das fazendas em pequena escala, de forma a melhorar o sustento dos agricultores. O problema é que os atuais modelos de abastecimento amplamente aplicados não incluem a melhoria dos meios de subsistência dos produtores / agricultores (SJAUW-KOEN-FA, et al., 2017).

Para Hanelt, Busse, e Kolbe, (2017), os sistemas de informação podem promover a transformação do negócio para sustentabilidade em grande escala, apoiando tecnologias verdes, criando assim soluções físico-digitais híbridas capazes de cumprir o desempenho organizacional, requisitos que contribuem para práticas comerciais sustentáveis. Esse é um caminho alternativo para a sustentabilidade corporativa, em vez de apenas contribuir para o "ecologização" das práticas comerciais, visando melhorar o impacto da organização no desempenho de inovações ambientais vantajosas, que por sua vez promove sua adoção.

A industrialização da agricultura expandiu-se muito nas décadas anteriores. As fazendas estão se desenvolvendo para fábricas de alta tecnologia que são caracterizados pela produção em larga escala e intensivo uso da tecnologia. As 
fazendas não só devem ser muito eficientes, mas também tem que cumprir padrões de alta qualidade e ambientais, e ainda deve se adaptar de forma flexível a condições impostas pelas mudanças do mercado (VERDOUW et al., 2015).

E importante redesenhar o modelo de negócio para que as empresas convencionais, possam integrar mais facilmente a sustentabilidade no seu negócio, e para novas empresas criativas poder projetar e buscar negócios sustentáveis, como sugerido por (STUBBS; COCKLIN, 2008) e (PORTER; KRAMER, 2011).

Diante dessa perspectiva, o estudo de Veldwisch, (2015) contribui para a discussão sobre modelos de negócios inclusivos como alternativas às aquisições de terras em larga escala, analisando um caso em que um produtor rural estrangeiro, dentro de um paradigma de investimento de impacto, adquiriu e reabilitou uma planta de processamento de arroz em Chokwe, Moçambique.

Já o estudo de Sjauw-Koen-Fa, et al., (2017) explora a aplicabilidade de um modelo sustentável de terceirização sustentável com uma lista de fatores críticos de sucesso, nos quais os objetivos comerciais e as perspectivas de responsabilidade social corporativa são combinados.

Assim, os arquétipos de modelos de negócios sustentáveis são introduzidos para descrever agrupamentos de mecanismos e soluções que podem contribuir para a construção do modelo de negócios para a sustentabilidade. A mira desses arquétipos é desenvolver uma linguagem comum que possa ser usada para acelerar a desenvolvimento de modelos empresariais sustentáveis em pesquisa e prática. Os arquétipos são: maximizar a eficiência de materiais e energia; criar valor a partir de "resíduos"; substituir por fontes renováveis e processos naturais; entregar funcionalidades em vez de propriedade; adotar um papel de administração; incentivar a suficiência; re-propósito do negócio para a sociedade / meio ambiente; e desenvolva a escala de soluções (BOCKEN et. al., 2014).

\section{METODOLOGIA}

Em um contexto de crescente produção acadêmica/científica em todos os campos, principalmente em relação ao agronegócio, face sua importância econômica, as pesquisas com um todo, assim como as revisões de literatura adquirem cada vez mais relevância, pois permitem um levantamento dos campos científicos, de forma a encontrar lacunas/gaps para contribuição na academia.

Nesse sentido, optamos por uma revisão bibliométrica para este trabalho. A pesquisa bibliométrica visa compreender a construção e o direcionamento das pesquisas acadêmicas sobre determinado fenômeno, neste caso, os estudos sobre Modelos de Negócios para Agronegócios na base Web of Science entre os anos de 1956 e 2017. Segundo Araújo (2006, p. 11), a bibliometria surge pela "necessidade do estudo e da avaliação das atividades de produção e comunicação científica". Nesse mesmo sentido, Chueke e Amatucci $(2015$, p 2) colocam:

Particularmente, no campo das ciências sociais aplicadas os estudos bibliométricos se concentram em examinar a produção de artigos em um determinado campo de saber, mapear as comunidades acadêmicas e identificar as redes de pesquisadores e suas motivações. Tais objetivos são tangibilizados por meio da criação de indicadores que buscam sumarizar as instituições e os autores mais prolíferos, os acadêmicos mais citados e as redes de coautorias (CHUEKE; AMATUCCI, 2015, p. 2) 
As pesquisas bibliométricas, tendo uma predominância quantitativa, de forma a aplicar técnicas estatísticas e matemáticas para avaliação da produção acadêmica, a bibliometria permite inferências qualitativas, que permite realizar uma análise de autores e obras mais referenciadas do campo em estudo e permite uma análise qualitativa das palavras-chave.

Nesse sentido, a análise bibliométrica consistem em uma frente de pesquisa, que são os artigos encontrados em uma determinada busca, os principais temas trabalhados dentro de um campo, os autores que mais publicam sobre um determinado tema, os países que lideram a pesquisa de um determinado assunto e permitem apontar, também, se existe alguma instituição de ensina central para uma temática, e a base intelectual, que mostra quais são as principais obras que sustentam uma determinada área de conhecimento e também quais os autores são referências centrais dentro de um campo.

Para Chueke e Amatucci $(2015$, p. 1), a bibliometria colabora na tarefa de sistematização das pesquisas realizadas em determinado campo e endereçar problemas investigados em pesquisa futuras. Nesse sentido, a pesquisa bibliométrica busca identificar o que está posto em um determinado campo de conhecimento e permite evidenciar tendências e lacunas para pesquisas futuras. Para tanto, estes dados fornecidos pelas bases de estudo podem ser considerados valiosos, de maneira que oferecem informações objetivas e compreensíveis sobre os fenômenos na academia e de maneira vasta para o campo analisado (Diem e Wolter 2013).

Para a organização dos procedimentos utilizados nesta revisão foi utilizado o framework proposto por Prado et al (2016), tendo sido realizadas pequenas adaptações referentes a este estudo. O framework apresentando no Quadro 1 mostra as etapas para a busca, seleção, organização e análise dos dados que constituirão o corpus deste estudo, o que possibilita a replicação do estudo, além de fornecer transparência para a pesquisa.

Quadro 1 - Framework de Pesquisa

\begin{tabular}{|c|c|c|c|}
\hline \multirow{4}{*}{1} & \multirow{4}{*}{$\begin{array}{c}\text { Operacionalização } \\
\text { da pesquisa }\end{array}$} & & Procedimento \\
\hline & & 1.1 & Escolha da(s) base(s) científica(s) ou periódicos \\
\hline & & 1.2 & Delimitação dos termos que representam o campo \\
\hline & & & Delimitação de outros termos para apurar os resultados \\
\hline \multirow{6}{*}{2} & \multirow{6}{*}{$\begin{array}{l}\text { Procedimentos de } \\
\text { busca } \\
\text { (filtros) }\end{array}$} & 2.1 & Title (termo do campo) AND topic (direcionamento) \\
\hline & & & Utilização de underline: expressão exata \\
\hline & & 2.3 & Filtro 1: Delimitação em somente artigos \\
\hline & & & Filtro 2: Todos os anos \\
\hline & & & Filtro 3: Todas as áreas \\
\hline & & & Filtro 4: Todos os idiomas \\
\hline \multirow{6}{*}{3} & \multirow{6}{*}{$\begin{array}{l}\text { Procedimentos de } \\
\text { seleção } \\
\text { (Banco de dados) }\end{array}$} & & Download das referências - software EndNote \\
\hline & & & Download das referências em formato planilha eletrônica \\
\hline & & 3.3 & Download das referências para utilização no CiteSpace \\
\hline & & 3.4 & Organização das referências no EndNote \\
\hline & & & Organização de matriz de análise em planilha eletrônica \\
\hline & & & Importação dos dados para softwares de análise \\
\hline \multirow{4}{*}{4} & \multirow{4}{*}{$\begin{array}{c}\text { Adequação e } \\
\text { organização } \\
\text { dos dados }\end{array}$} & & Eliminação dos artigos duplicados no banco de dados \\
\hline & & 4.2 & Eliminação de artigos por meio de leitura flutuante \\
\hline & & 4.3 & Eliminação por meio da análise da polissemia dos termos \\
\hline & & 4.4 & Busca dos artigos completos em .pdf \\
\hline
\end{tabular}




\begin{tabular}{|l|l|l|l|}
\hline \multirow{4}{*}{5} & 5.1 & Análise do volume das publicações e tendências temporais \\
\cline { 3 - 4 } & $\mathbf{5 . 2}$ & Análise das referências e artigos mais citados \\
\cline { 3 - 4 } & $\mathbf{5 . 3}$ & Análise do país de origem \\
\cline { 3 - 4 } $\begin{array}{c}\text { Análise da } \\
\text { produção } \\
\text { científica }\end{array}$ & $\mathbf{5 . 4}$ & Análise dos periódicos \\
\cline { 3 - 4 } & $\mathbf{5 . 5}$ & Análise da autoria e coautoria \\
\cline { 3 - 4 } & $\mathbf{5 . 6}$ & Análise das categorias (áreas) das publicações \\
\cline { 3 - 4 } & $\mathbf{5 . 7}$ & Análise das palavras-chave \\
\cline { 3 - 4 } & $\mathbf{5 . 8}$ & Descrição, estudo das relações e tendências \\
\hline
\end{tabular}

Fonte: Prado et al (2016).

Conforme demonstrado no Quadro 1, para o início da bibliometria faz-se necessário definir as etapas da busca será realizada. Para a base de dados, foi selecionada a base Web of Science (coleção principal) da Thomson Reuters Scientific por ser considerada uma das mais completas, de forma que, a escolha por uma única base de dados está relacionada com a padronização das informações (PRADO et al., 2016) e a escolha da Web of Science justifica-se por sua relevância, abrangência e confiabilidade (PINTO; SERRA; FERREIRA, 2014). Outro fator relevante, a Web of Science permite exportações das buscas e referências para a utilização via software CiteSpace, o software mais utilizado para realização da bibliometria (CHEN, 2006) e que será utilizado neste trabalho.

Segundo Chen (2006), o CiteSpace permite a construção das duas etapas da pesquisa bibliométrica: o Research front (os trabalhos mais citados de um campo científico), e a Intellectual base (as citações e cocitações na literatura científica). Estes conceitos são importantes para demonstrar tendências e padrões na literatura científica de uma determinada área de conhecimento (CHEN, 2006).

Adiante, para a realização da busca utilizamos os termos "Business Model", "Agrobusiness" e "Agribusiness", entre aspas, de forma a aparecer o termo inteiro nas buscas pelo título ou no corpo dos artigos. Foram considerados todos os anos da base, de 1956 até 2017 - último ano completo - assim como todos os idiomas e categorias, restringindo a busca aos artigos científicos presentes na base e excluindo trabalhos como livros, anais de conferências, entre outros, resultando em um total de 1905 artigos. Adiante, para utilização do CiteSpace foi realizado o download das referências dos artigos, que foram exportadas em formato de planilha eletrônica para tabulação e também em formato de texto para utilização do software CiteSpace (CHEN, 2006).

A análise da frente de pesquisa consistiu em analisar quantitativamente e qualitativamente os 1.905 trabalhos encontrados na busca. Nesse sentido, realizouse uma análise da frequência de publicações por ano, com o intuito de verificar o seu início e se existem tendências no volume, de forma a identificar os períodos de maior publicação ao longo dos anos. Foram analisados, através das informações levantadas na Web of Science com a utilização do Software CiteSpace, as palavras-chaves, pois elas podem nos indicar as principais temáticas dentro de um campo de conhecimento. Por sua vez, a análise da Intellectual base de uma frente de pesquisa nos mostra as citações e cocitações, ou seja, quais são os trabalhos e autores referenciados pelos artigos (CHEN, 2006).

\section{ANÁLISE E DISCUSSÃO DOS RESULTADOS}

Nesta etapa são apresentados os resultados da análise descritiva, que contempla os dados bibliométricos da pesquisa. É possível verificar que as publicações na área de modelos de agronegócios se iniciaram em 1956, seguido por uma lacuna nos anos seguintes e tendo a segunda publicação 8 anos depois, em 
1964. Os anos seguintes se destacaram por um acúmulo das publicações na área, mas ainda sem um destaque maior, o que denota pouco interesse para as pesquisas na área até então. É possível notar um maior avanço no número de pesquisas realizadas a partir do ano de 1994, com 14 artigos científicos. A Figura 1 representa a produção acadêmica, ao longo dos anos de forma mais detalhada.

Figura 1 - Publicações por Ano

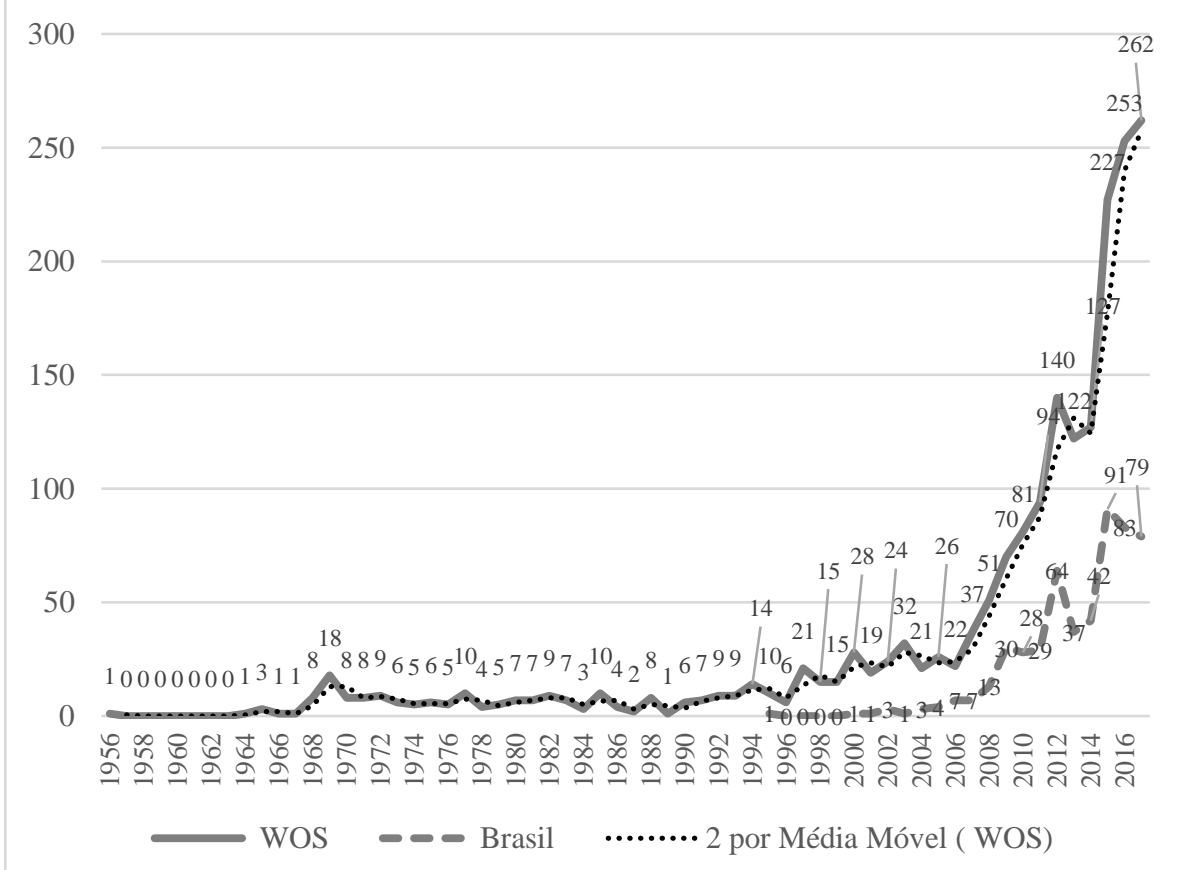

Fonte: Dados da Pesquisa.

Na Figura 1 é possível perceber que de 1994 a 2006 as publicações, apesar do leve crescimento, ainda se encontram de forma estável, sempre abaixo dos 30 artigos científicos por ano, com exceção para o ano de 2003, com 32 artigos. Do ano de 2007 em diante, ano que totalizou 37 artigos científicos, as publicações tiveram um crescimento exponencial, com apenas os anos de 2013 e 2014 apresentando queda na produção acadêmica, tendo 122 e 127 artigos respectivamente. Dessa forma, é possível notar que foi dada maior importância a temática a partir do ano de 1994, mas o tema se tornou realmente relevante a partir do ano de 2007, conforme apresentado pela linha de tendência por média móvel com base em dois períodos, onde a linha não apresenta declínio nos períodos posteriores a 2007.

Em relação ao Brasil, sua primeira publicação só ocorreu em 1995, sua segunda publicação apenas em 2000 , e seu crescimento se deu a partir de 2006 , com 7 artigos. Apesar do começo tardio, é possível notar uma grande participação brasileira na produção acadêmica da temática, envolvendo modelos de negócios para agronegócios, de um total de 1905 artigos encontrados, 523 são brasileiros, o que corresponde a $27,5 \%$ das publicações na área. 
Figura 2 - Citações por Ano

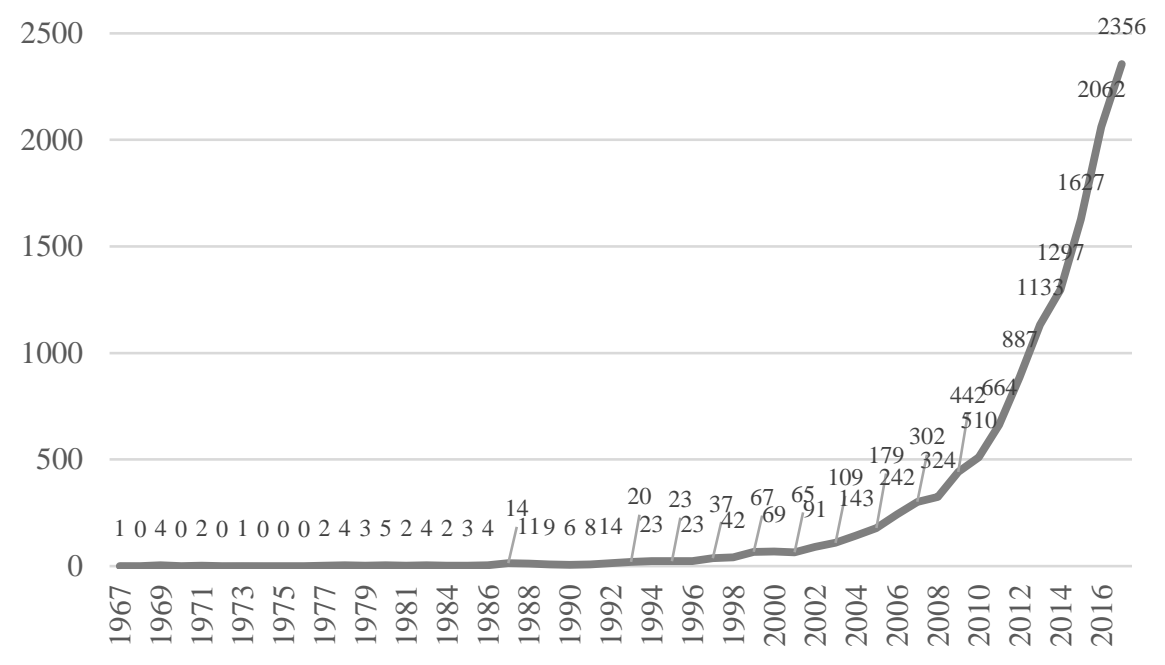

Fonte: Dados da Pesquisa.

A Figura 2 apresenta as citações por ano dentro da temática de modelos de negócios para o agronegócio, tendo sua primeira citação sido realizado em 1964, 8 anos após a publicação do primeiro artigo científico na área. É possível notar um maior crescimento das citações a partir do ano de 1994, corroborando com a Figura 1, onde é apresentado um maior crescimento das publicações na área. A partir do ano de 2004 as citações já ultrapassaram a cada 3 dígitos, o que também corrobora com as publicações na área, tendo seu crescimento a partir de 2007 , conforme linha de tendência por média móvel apresentada acima.

Figura 3 - Principais Autores

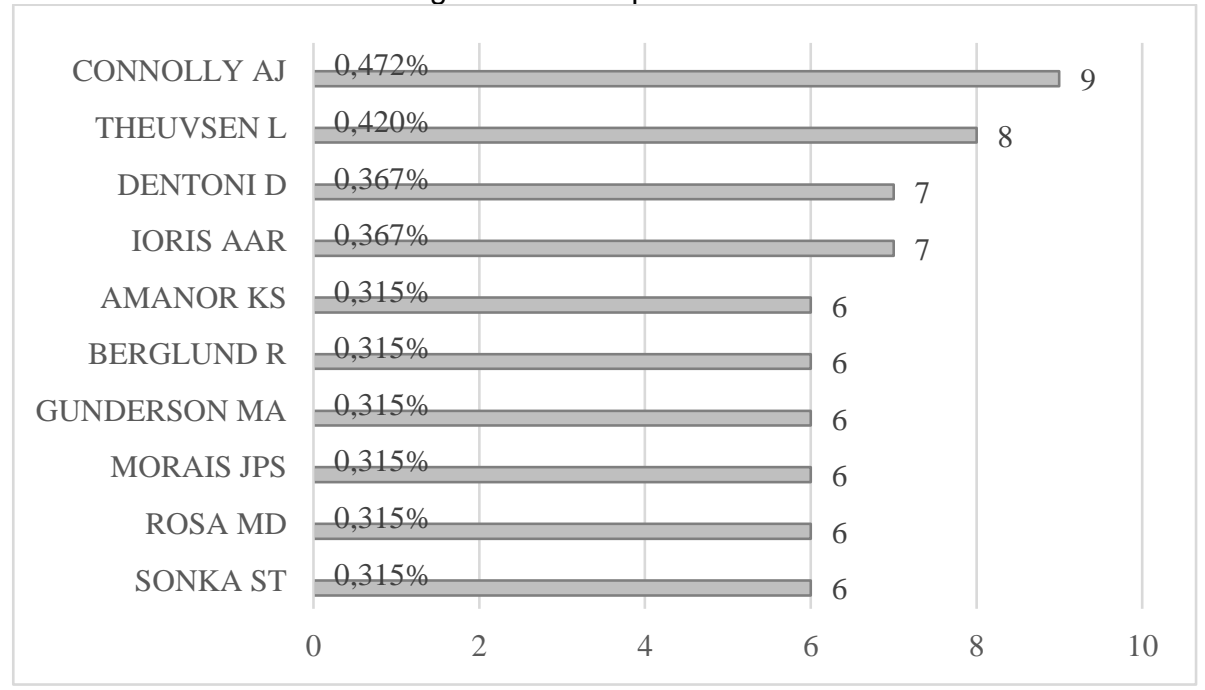

Fonte: Dados da Pesquisa. 
Os 10 principais autores na área, conforme apontado pela Figura 3, correspondem a 67 artigos, do total de 1905 artigos científicos publicados na área, não sendo possível determinar uma elite acadêmica em relação a temática, mas destacam-se os autores Connolly, 9 artigos, Theuvsen, 8 artigos, e Dentoni e loris, ambos com 7 artigos científicos, seguidos de uma maior sucessão de autores com 6 artigos na área.

Para tanto, é importante destacar a contribuição dos autores João Paulo Saraiva Morais e Morsyleide de Freitas Rosa, ambos com 6 artigos científicos, sendo autores brasileiros que estão apresentados entre os 10 autores com mais contribuição para as publicações da área.

Figura 4 - Autores mais citados

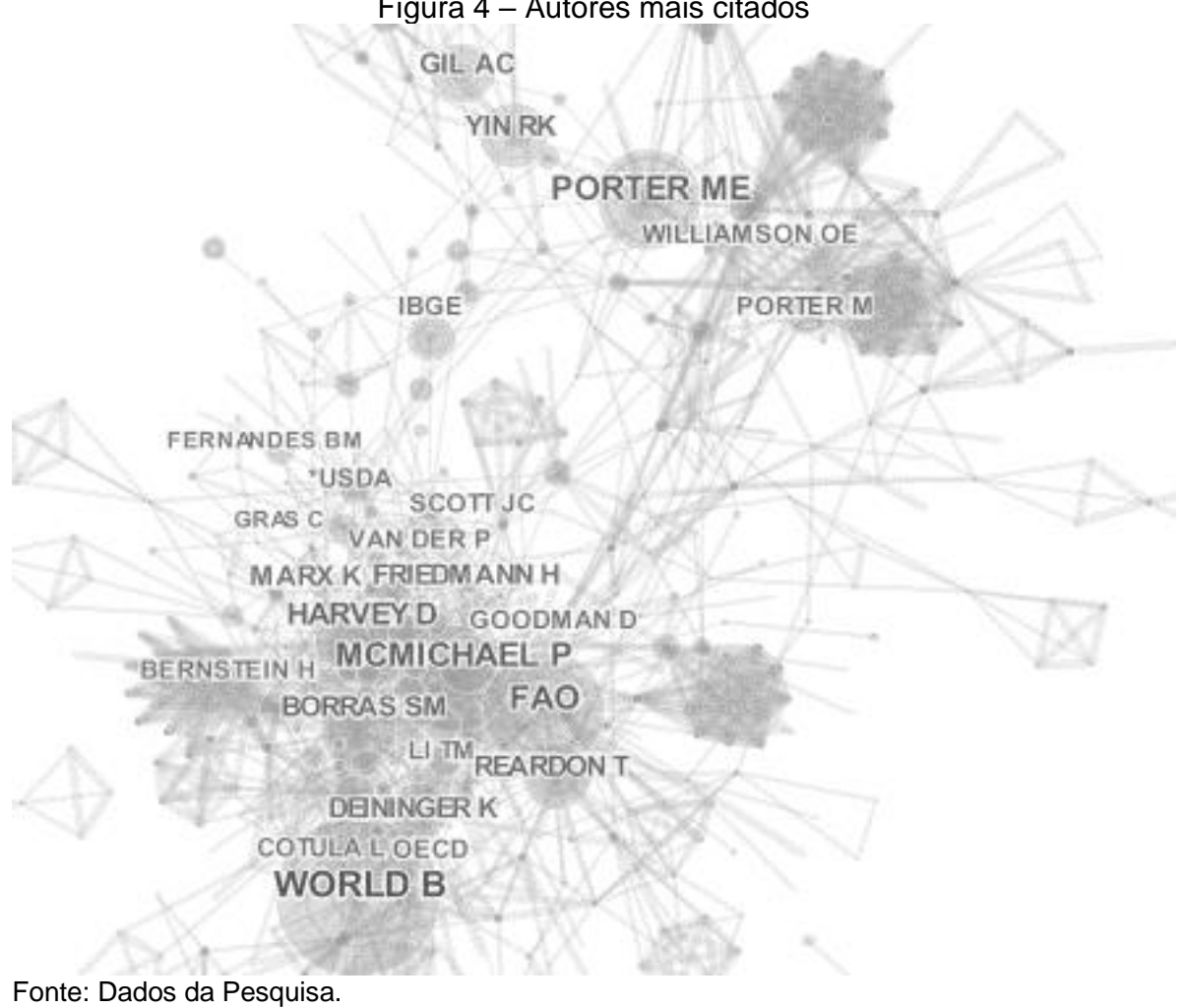

A análise via CiteSpace mostrada na Figura 4 aponta para os autores mais citados nos artigos, com um destaque para Porter e para a fonte consultada IBGE, o Instituto Brasileiro de Geografia e Estatística, que como base brasileira pesquisada, reforça o destaque do Brasil neste campo de pesquisa. Dentre os principais autores apontados na Figura 3, nenhum deles consta dentre as fontes apresentadas na análise via CiteSpace. 
Figura 5 - Referências mais citadas

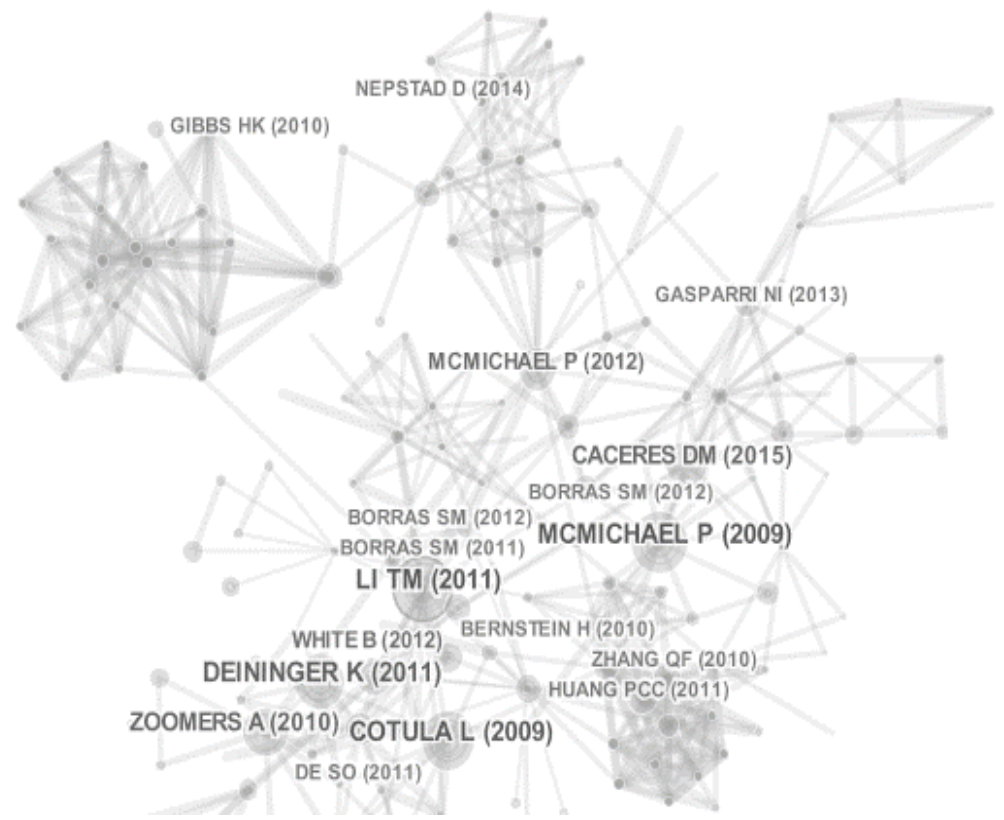

Fonte: Dados da Pesquisa.

A Figura 5 apresenta as principais referências presentes nos artigos pesquisados, onde o mesmo, novamente, não corrobora com a Figura 3, que aponta os principais autores da área, mas aponta para a referência principal de Porter nestas pesquisas, apontado acima em trabalhos, principalmente, nos anos de 2009 e 2012.

Figura 6 - Publicações por Países

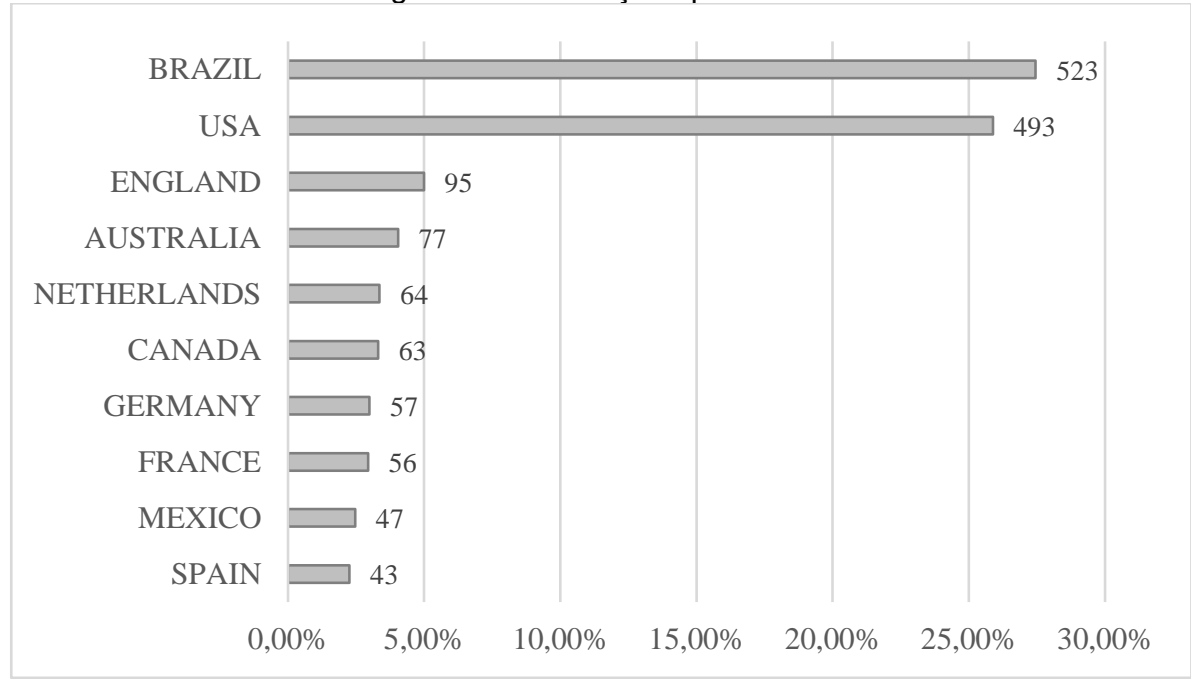

Fonte: Dados da Pesquisa. 
Dessa forma, a Figura 6 apresenta a quantidade de publicações por países, dando a importância ao Brasil, com 523 artigos científicos na área, um total de 27,5\% das publicações sobre a temática sendo proveniente da academia brasileira. É importante destacar também a posição dos Estados Unidos, com 493 publicações, $25,8 \%$ das publicações da área, sendo que, dessa forma, os dois países, Brasil e EUA, concentram um total de $53,3 \%$ das publicações sobre a temática de modelos de negócios para agronegócios.

Figura 7 - Principais Agências Financiadoras

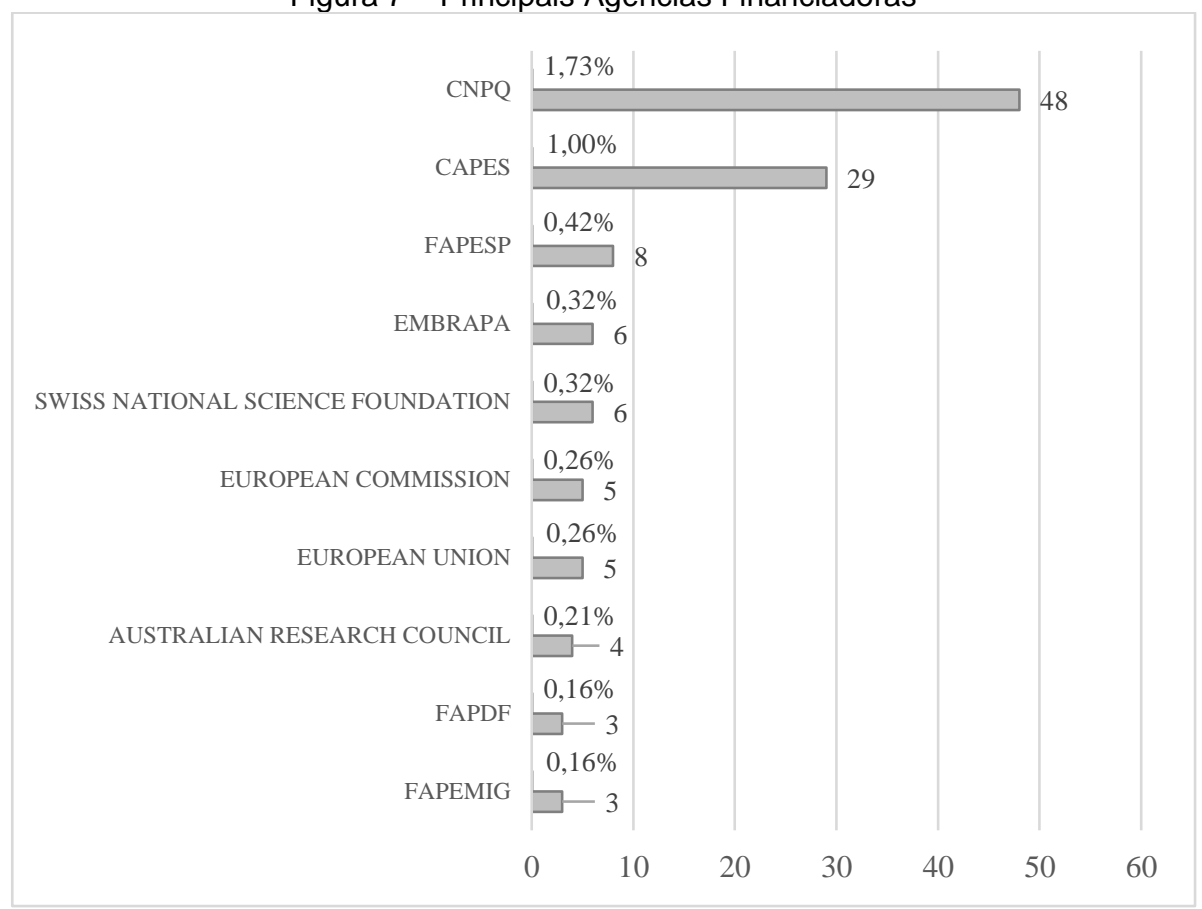

Fonte: Dados da Pesquisa.

Dentre as principais agências financiadoras, conforme apresentado na Figura 7, 6 agências de fomento são brasileiras, o que demonstra a preocupação da academia brasileira em relação a temática. Vale ressaltar que a quantidade de agências de fomento que financiam pesquisas na área é grande, um total de 619 agências de fomento foram encontradas para as pesquisas mundiais, o que denota preocupação de governos com pesquisas na área, e que as 6 brasileiras (Conselho Nacional de Desenvolvimento Científico e Tecnológico - CNPQ, Coordenação de Aperfeiçoamento de Pessoal de Nível Superior - CAPES, Fundação de Amparo à Pesquisa do Estado de São Paulo - FAPESP, Empresa Brasileira de Pesquisa Agropecuária - EMBRAPA, Fundação de Apoio à Pesquisa do Distrito Federal - FAPDF e Fundação de Amparo à Pesquisa do Estado de Minas Gerais - FAPEMIG) concentram um total de 3,78\% das pesquisas mundiais sobre a temática. 
Figura 8 - Principais Journals

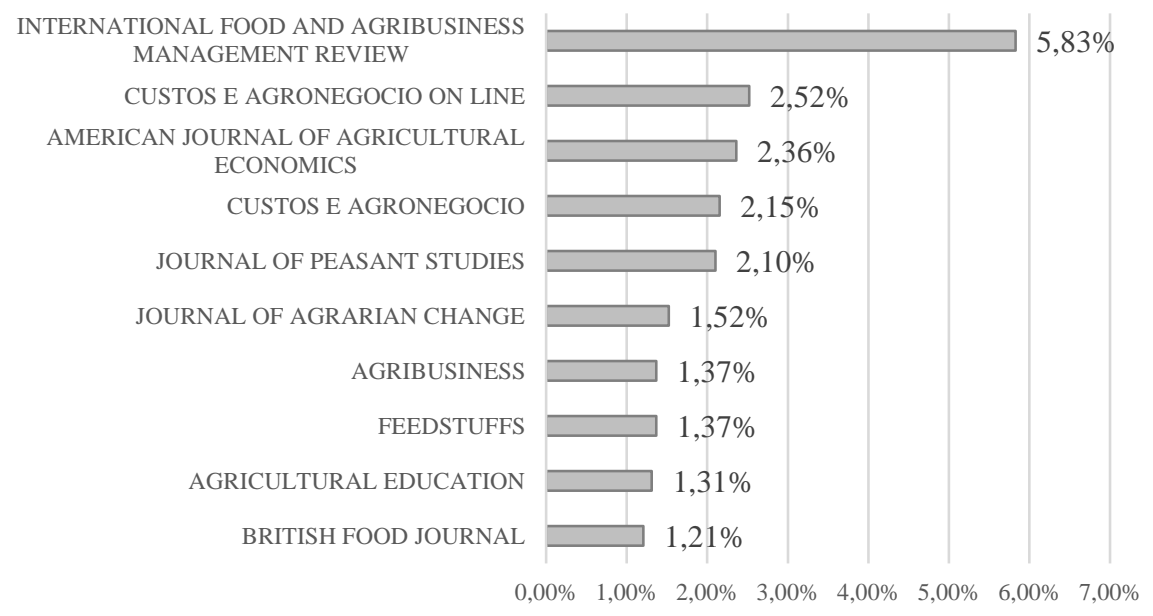

Fonte: Dados da Pesquisa.

Dentre os principais Journals da área, destaca-se o International Food and Agribusiness Management Review, com 5,83\% das publicações na área, o maior periódico em quantidade de publicações. Mas há de se considerar a relevância dos periódicos Custos e Agronegócios e Custos e Agronegócios Online, um periódico brasileiro em duas plataformas distintas, e que aparecem entre os 10 principais periódicos da área. Esta informação em si é mais um reforço para o pensamento sobre a grande relevância que é dada pela academia a área de agronegócios e, consecutivamente, a seus modelos de negócios.

Figura 9 - Principais Áreas de Pesquisa

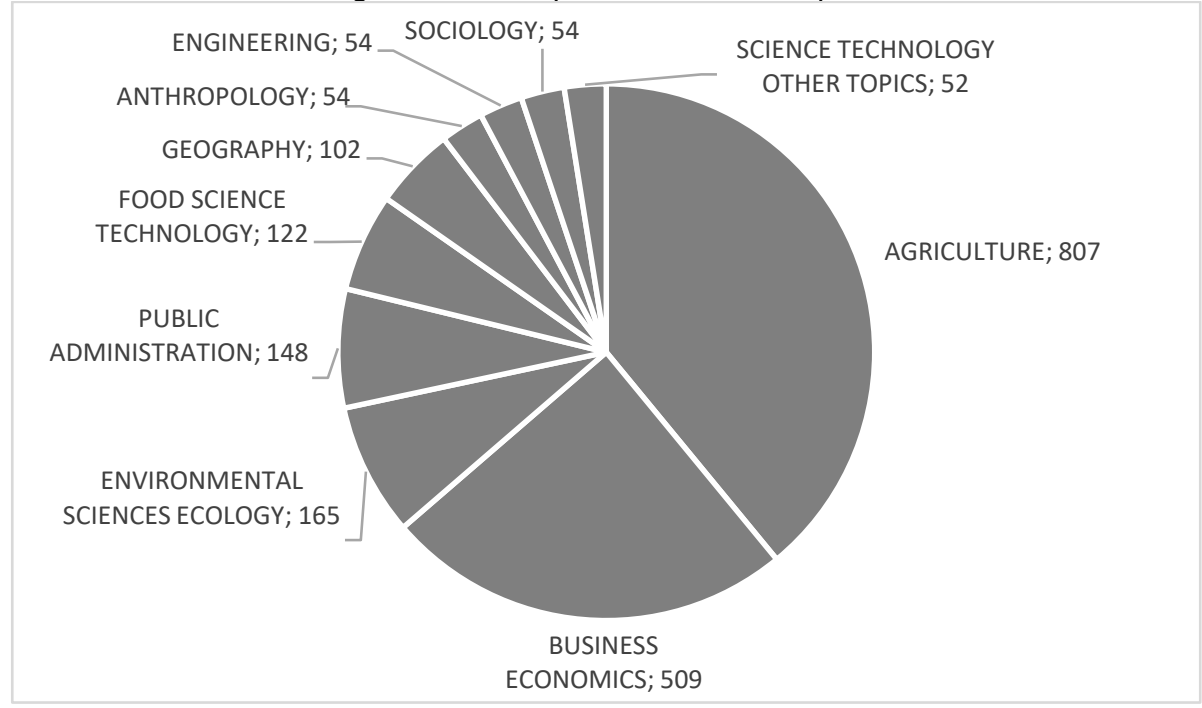

Fonte: Dados da Pesquisa. 
Dentre as principais áreas de pesquisa, as primeiras posições são relevantes a Agriculture e Business Economics, que são as temáticas fontes dessa pesquisa. Porém é importante destacar a terceira colocação para as pesquisas de Environmental Sciences Ecology, o que denota uma preocupação das em relação as pesquisas ambientais. Essa preocupação em si se torna uma possibilidade de aprofundamentos para pesquisas futuras, visto que é importante aprofundar a relação existente entre os modelos de agronegócios que envolvem as questões ambientais, buscando compreender o motivo destas pesquisas, se por uma busca de maior sustentabilidade nos agronegócios ou se por questões restritivas do ambiente.

$\mathrm{Na}$ Tabela 1 são apresentados os artigos mais citados nesta pesquisa.

Tabela 1 - Artigos mais citados na Web of Science

\begin{tabular}{|c|c|c|c|c|}
\hline Artigo & Autor & Ano & Journal & Citações \\
\hline $\begin{array}{l}\text { An overview of APSIM, a } \\
\text { model designed for farming } \\
\text { systems simulation }\end{array}$ & $\begin{array}{l}\text { Keating, BA; } \\
\text { Carberry, PS; } \\
\text { Hammer, GL; et al. }\end{array}$ & 2003 & $\begin{array}{l}\text { European Journal } \\
\text { of Agronomy }\end{array}$ & 1106 \\
\hline $\begin{array}{l}\text { Global change and } \\
\text { eutrophication of coastal } \\
\text { Waters }\end{array}$ & $\begin{array}{l}\text { Rabalais, NN; } \\
\text { Turner, RE; Diaz, } \\
\text { RJ; et al. }\end{array}$ & 2009 & $\begin{array}{l}\text { Ices Journal of } \\
\text { Marine Science }\end{array}$ & 307 \\
\hline $\begin{array}{l}\text { Road paving, fire regime } \\
\text { feedbacks, and the future of } \\
\text { Amazon forests }\end{array}$ & $\begin{array}{l}\text { Nepstad, D; } \\
\text { Carvalho, G; } \\
\text { Barros, AC; et al. }\end{array}$ & 2001 & $\begin{array}{l}\text { Forest Ecology } \\
\text { and Management }\end{array}$ & 290 \\
\hline $\begin{array}{l}\text { From farm to table: The } \\
\text { organic vegetable commodity } \\
\text { chain of northern California }\end{array}$ & $\begin{array}{l}\text { Buck, D; Getz, C; } \\
\text { Guthman, J }\end{array}$ & 1997 & Sociologia Ruralis & 191 \\
\hline $\begin{array}{l}\text { The trouble with 'organic lite' } \\
\text { in California: A rejoinder to } \\
\text { the 'conventionalisation' } \\
\text { debate }\end{array}$ & Guthman, J & 2004 & Sociologia Ruralis & 174 \\
\hline $\begin{array}{l}\text { The agroecological } \\
\text { revolution in Latin America: } \\
\text { rescuing nature, ensuring } \\
\text { food sovereignty and } \\
\text { empowering peasants }\end{array}$ & $\begin{array}{l}\text { Altieri, MA; Manuel } \\
\text { Toledo, V }\end{array}$ & 2011 & $\begin{array}{l}\text { Journal of Peasant } \\
\text { Studies }\end{array}$ & 170 \\
\hline $\begin{array}{l}\text { The international political } \\
\text { economy of the global land } \\
\text { rush: A critical appraisal of } \\
\text { trends, scale, geography and } \\
\text { drivers }\end{array}$ & Cotula, L & 2012 & $\begin{array}{l}\text { Journal of Peasant } \\
\text { Studies }\end{array}$ & 156 \\
\hline $\begin{array}{l}\text { Committed carbon } \\
\text { emissions, deforestation, and } \\
\text { community land conversion } \\
\text { from oil palm plantation } \\
\text { expansion in West } \\
\text { Kalimantan, Indonesia } \\
\end{array}$ & $\begin{array}{l}\text { Carlson, KM.; } \\
\text { Curran, LM.; } \\
\text { Ratnasari, D; et al. }\end{array}$ & 2012 & $\begin{array}{l}\text { Proceedings of the } \\
\text { National Academy } \\
\text { of Sciences of fthe } \\
\text { United States of } \\
\text { America }\end{array}$ & 134 \\
\hline $\begin{array}{l}\text { Processes of inclusion and } \\
\text { adverse incorporation: oil } \\
\text { palm and agrarian change in } \\
\text { Sumatra, Indonesia }\end{array}$ & McCarthy, JF & 2010 & $\begin{array}{l}\text { Journal of Peasant } \\
\text { Studies }\end{array}$ & 123 \\
\hline $\begin{array}{l}\text { A food regime analysis of the } \\
\text { 'world food crisis' }\end{array}$ & McMichael, P & 2009 & $\begin{array}{l}\text { Agriculture and } \\
\text { Human Values }\end{array}$ & 119 \\
\hline
\end{tabular}

Fonte: Dados da Pesquisa. 
Dentre os 10 principais artigos apresentados na Tabela 1, é de destaque para o artigo "An overview of APSIM, a model designed for farming systems simulation", de Keating, BA; Carberry, PS; Hammer, GL; et al. (2003), publicado pelo European Journal of Agronomy, e que possui 1.106 citações acadêmicas até o momento desta pesquisa, um número impressionante se comparado com as 307 citações do segundo artigo mais citado.

O artigo de Keating, Carberry e Hammer (2003) trata de um Simulador de Sistemas de Produção Agrícola (APSIM), desenvolvida para simular o processo biofísico nos sistemas agrícolas, em particular quando há interesse nos resultados econômicos e ecológicos da prática de gestão em face do risco climático. $\mathrm{O}$ artigo apresenta a ampla gama de aplicações da APSIM, incluindo suporte para tomada de decisões na fazenda, design de sistemas agrícolas para a produção ou objetivos de gerenciamento de recursos, avaliação de previsão de valor sazonal, análise de questões de cadeia de suprimentos nas atividades do agronegócio, desenvolvimento de diretrizes de gerenciamento de resíduos, avaliação de riscos para a elaboração de políticas governamentais e um guia para pesquisa e atividade educacional.

O artigo de Rabalais et. al. (2003), aponta os efeitos cumulativos da mudança global, incluindo mudanças climáticas, aumento da população e industrialização e agro-negócios mais intensos, tendo como resultado os sintomas da eutrofização, como flores de algas nocivas e prejudiciais, redução da qualidade da água, perda de habitat e recursos naturais, e gravidade da hipoxia (depleção de oxigênio) e sua extensão em estuários e águas costeiras aumentarão. Segundo os autores, as mudanças climáticas globais provavelmente resultarão em maiores temperaturas da água, estratificação mais forte e aumento de entradas de água doce e nutrientes para as águas costeiras em muitas áreas do globo.

Nepstad et. al. (2001) por outro lado, em seu artigo, apresenta o fogo como a maior ameaça para as florestas da Amazônia. Para os autores, a magnitude desta ameaça é ampliada por três laços que impulsionam a expansão do incêndio florestal na região: (1) O fogo promove a seca e, portanto, mais fogo, liberando fumaça para a atmosfera, reduzindo assim as chuvas; (2) O fogo aumenta a susceptibilidade das florestas à queima recorrente ao matar árvores, permitindo que a luz solar penetre no interior da floresta e aumente a carga de combustível no chão da floresta; e (3) Finalmente, os incêndios também se autoperpetam pela queima de sistemas agrícolas e florestais, desencorajando os proprietários de terra de fazerem esses investimentos sensíveis ao fogo em suas terras, o que lhes permitiria ultrapassar sua dependência do fogo como uma ferramenta de gerenciamento.

Além dos 3 principais artigos acima descritos e que trazem uma preocupação com questões ambientais, destaca-se também a participação de autores brasileiros dentre os 10 artigos mais citados na área. Os artigos "Road paving, fire regime feedbacks, and the future of Amazon forests", dos autores Nepstad, D; Carvalho, G; Barros, AC; et al. (2001), publicado pela Forest Ecology and Management, e o artigo "Committed carbon emissions, deforestation, and community land conversion from oil palm plantation expansion in West Kalimantan, Indonesia", dos autores Carlson, Kimberly M.; Curran, Lisa M.; Ratnasari, Dessy; et al. (2012), publicado pelo Proceedings of the National Academy of Sciences of the United States of America, ambos que possui autores brasileiros na sua construção.

\section{CONSIDERAÇÕES FINAIS}

O presente estudo teve como objetivo mapear o estado da pesquisa em desenvolvimento de modelo de negócios para o agronegócio, a fim de proporcionar uma melhor compreensão da estrutura deste campo de pesquisa e dos principais 
atores envolvidos. Especificamente, procurou-se apresentar as tendências de publicação nesse campo, as categorias (áreas) das publicações, as palavras chaves e países centrais, os artigos, periódicos e autores mais relevantes, as estruturas de cocitação e coautoria dos mesmos.

O artigo investigou a evolução das publicações sobre o desenvolvimento de modelo de negócio para o agronegócio, identificando um crescimento significativo a partir de 2007. Entre os principais autores da área, não foi possível determinar uma elite acadêmica em relação a temática, mas os autores que se destacam são Connolly, Theuvsen, Dentoni, loris, Morais e Rosa. Ao comparar os países mais citados, identifica-se o Brasil e Estados Unidos com maior volume de publicações sobre a temática, destaque especial para o Brasil que lidera em primeiro lugar no volume de publicações sobre a temática.

$\mathrm{Na}$ análise das principais agências financiadoras, se destacam CNPQ e CAPES com maior volume de artigos publicados e financiados por elas. Dentre os principais Journals da área, destaca-se o International Food and Agribusiness Management Review. As principais áreas de pesquisa são Agriculture e Business Economics, dentre as temáticas que foram fonte dessa pesquisa.

Os resultados mostram a grande participação do Brasil nas publicações sobre modelos de negócio para o agronegócio. O país apresenta o maior número de publicações dentro da busca realizada. Além disso, o Brasil apresenta periódicos entre os periódicos com maior número de artigos publicados, sendo referência no assunto. Mais que isso, possui as principais agências financiadoras de pesquisas no assunto. Portanto, o Brasil pode ser considerado referência no assunto, o que condiz com sua condição atual no agronegócio. Isso demonstra que o país produz pesquisas com 0 objetivo de desenvolver seu agronegócio.

Como limitação, destacamos que a revisão bibliométrica aqui realizada foi internacional e pautada em uma única base. Nesse sentido, recomenda-se revisões de literatura nacionais sobre o tema. Além disso, recomenda-se o uso de outras bases de dados. Por fim, recomenda-se pesquisa empíricas sobre modelos de negócios voltados para o agronegócio.

\section{REFERÊNCIAS}

ARBAGE, A. P. Fundamentos de Economia Rural. Chapecó: Argos, 2012.

BAINES, T., LIGHTFOOT, H., EVANS, S., E NEELY, A. State-of-the-art in productservice systems. Proceedings of the Institution of Mechanical Engineers, Part B: Journal of Engineering Manufacture, 221 (10), p.1543-1552, 2007.

BEATTIE, V., SMITH, S. Value creation and business models: Refocusing the intellectual capital debate. The British Accounting Review, v. 45, 4, p. 243-254, 2013.

BOCKEN, N. M. P., SHORT, S., RANA, P., E EVANS, S. A literature and practice review to develop sustainable business model archetypes. Journal of Cleaner Production, 65: p.42-56, 2014.

BOCKEN, N., SHORT, S., RANA, P., E EVANS, S. A value mapping tool for sustainable business modelling. Corporate Governance, 13 (5), p.482-497, 2013.

CHEN, C. CiteSpace II: Detecting and visualizing emerging trends and 37ransiente patterns in scientific literature. JASIST, v, 57, n. 3, p. 359-377. 2006. 
COKER, A. A. A., et al. Gender Differentials among Subsistence Rice Farmers and Willingness to undertake Agribusiness in Africa: Evidence and Issues from Nigeria. African Development Review-Revue Africaine De Developpement 29: p.198-212, 2017.

HANELT, A., BUSSE, S., E KOLBE, L. M. Driving business transformation toward sustainability: exploring the impact of supporting IS on the performance contribution of eco-innovations. Information Systems Journal 27(4): p.463-502, 2017.

KEATINGM B. A. et. Al. An overview of APSIM, a model designed for farming systems simulation. European Journal of Agronomy, v. 18, ed. 3-4, p. 267-288, 2003.

NEPSTAD, D. et. al. Road paving, fire regime feedbacks, and the future of Amazon forests. Forest Ecology and Management, v. 154, ed. 3, p. 395-407, 2001.

OSTERWALDER, Alexander; PIGNEUR, Yves. Value proposition design. United States of America: Wiley Library, 2014.

PINTO, C. F.; SERRA, F. R.; FERREIRA, M. P. A bibliometric study on culture research in International Business. BAR, v. 11, n. 3, p. 340-363, 2014.

PORTER, M., KRAMER, M. Creating Shared Value. Harvard Business Review, February, 2011.

PRADO, J. W.; CASTRO ALCÂNTARA, V.; MELO CARVALHO, F.; VIEIRA, K. C.; MACHADO, L. K.; TONELLI, D. F. Multivariate analysis of credit risk and bankruptcy research data: a bibliometric study involving different knowledge fields (1968--2014). Scientometrics, v. 106, ed. 3, p. 1007-1029, 2016.

RABALAIS, N. N. et. al. Global change and eutrophication of coastal Waters. Ices Journal of Marine Science, v. 66, ed. 7, p. 1528-1537, 2009.

SJAUW-KOEN-FA, A. R., et al. Exploring the applicability of a sustainable smallholder sourcing model in the black soybean case in Java. International Food and Agribusiness Management Review 20(5): p.709-727, 2017.

SOMMER, A. Managing green business model transformations. Springer Verlag, Berlin Heidelberg, 2012.

STUBBS, W., COCKLIN, C. Conceptualizing a "Sustainability Business Model". Organization \& Environment, 21(2), p. 103-127, 2008.

VELDWISCH, G. J. Contract farming and the reorganisation of agricultural production within the Chokwe Irrigation System, Mozambique. Journal of Peasant Studies 42(5), p. 1003-1028, 2015.

VERDOUW, C. N., et al. ERP in agriculture: Lessons learned from the Dutch horticulture. Computers and Electronics in Agriculture 114, p.125-133, 2015.

ZOTT, C., AMIT, R., MASSA, L. The Business Model: Recent Developments and Future Research. Journal of Management, 37(4), p.1019-1042, 2011. 